\title{
General-Appell Polynomials within the Context of Monomiality Principle
}

\author{
Subuhi Khan and Nusrat Raza \\ Department of Mathematics, Aligarh Muslim University, Aligarh 202002, India \\ Correspondence should be addressed to Subuhi Khan; subuhi2006@gmail.com
}

Received 22 September 2012; Accepted 6 December 2012

Academic Editor: Jacques Liandrat

Copyright ( 2013 S. Khan and N. Raza. This is an open access article distributed under the Creative Commons Attribution License, which permits unrestricted use, distribution, and reproduction in any medium, provided the original work is properly cited.

\begin{abstract}
A general class of the 2-variable polynomials is considered, and its properties are derived. Further, these polynomials are used to introduce the 2-variable general-Appell polynomials (2VgAP). The generating function for the $2 \mathrm{VgAP}$ is derived, and a correspondence between these polynomials and the Appell polynomials is established. The differential equation, recurrence relations, and other properties for the $2 \mathrm{VgAP}$ are obtained within the context of the monomiality principle. This paper is the first attempt in the direction of introducing a new family of special polynomials, which includes many other new special polynomial families as its particular cases.
\end{abstract}

\section{Introduction and Preliminaries}

The Appell polynomials are very often found in different applications in pure and applied mathematics. The Appell polynomials [1] may be defined by either of the following equivalent conditions: $\left\{A_{n}(x)\right\}\left(n \in \mathbb{N}_{0}\right)$ is an Appell set $\left(A_{n}\right.$ being of degree exactly $n$ ) if either,

(i) $(d / d x) A_{n}(x)=n A_{n-1}(x)\left(n \in \mathbb{N}_{0}\right)$ or

(ii) there exists an exponential generating function of the form

$$
A(t) \exp (x t)=\sum_{n=0}^{\infty} A_{n}(x) \frac{t^{n}}{n !},
$$

where $A(t)$ has (at least the formal) expansion:

$$
A(t)=\sum_{n=0}^{\infty} A_{n} \frac{t^{n}}{n !} \quad\left(A_{0} \neq 0\right) .
$$

Roman [2] characterized Appell sequences in several ways. Properties of Appell sequences are naturally handled within the framework of modern classical umbral calculus by Roman [2]. We recall the following result [2, Theorem 2.5.3], which can be viewed as an alternate definition of Appell sequences.
The sequence $A_{n}(x)$ is Appell for $g(t)$, if and only if

$$
\frac{1}{g(t)} \exp (x t)=\sum_{n=0}^{\infty} A_{n}(x) \frac{t^{n}}{n !},
$$

where

$$
g(t)=\sum_{n=0}^{\infty} g_{n} \frac{t^{n}}{n !} \quad\left(g_{0} \neq 0\right) .
$$

In view of (1) and (3), we have

$$
A(t)=\frac{1}{g(t)} .
$$

The Appell class contains important sequences such as the Bernoulli and Euler polynomials and their generalized forms. Some known Appell polynomials are listed in Table 1.

We recall that, according to the monomiality principle $[15$, 16], a polynomial set $\left\{p_{n}(x)\right\}_{n \in \mathbb{N}}$ is "quasimonomial", provided there exist two operators $\widehat{M}$ and $\widehat{P}$ playing, respectively, the role of multiplicative and derivative operators, for the family of polynomials. These operators satisfy the following identities, for all $n \in \mathbb{N}$ :

$$
\begin{aligned}
& \widehat{M}\left\{p_{n}(x)\right\}=p_{n+1}(x), \\
& \widehat{P}\left\{p_{n}(x)\right\}=n p_{n-1}(x) .
\end{aligned}
$$




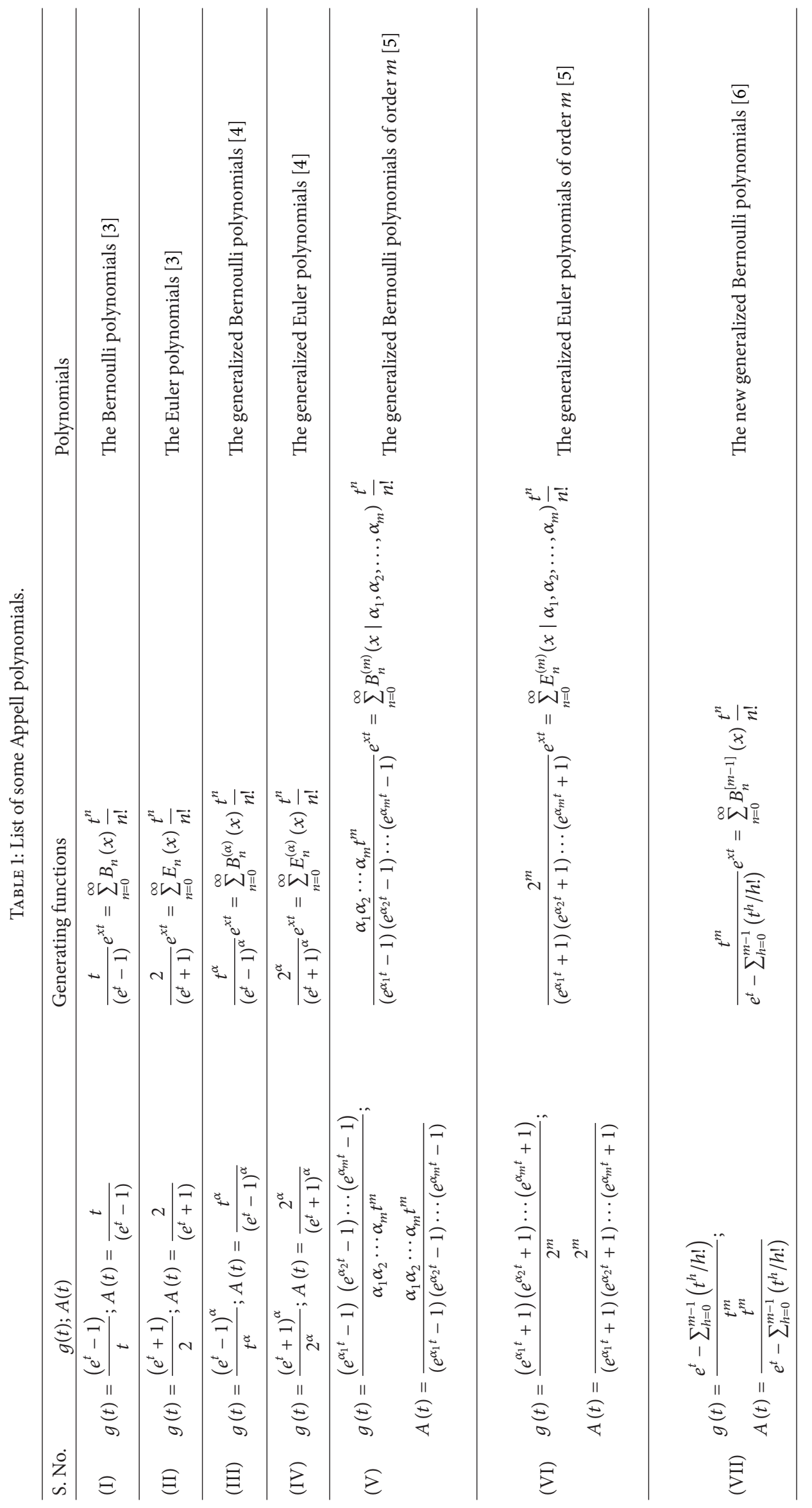




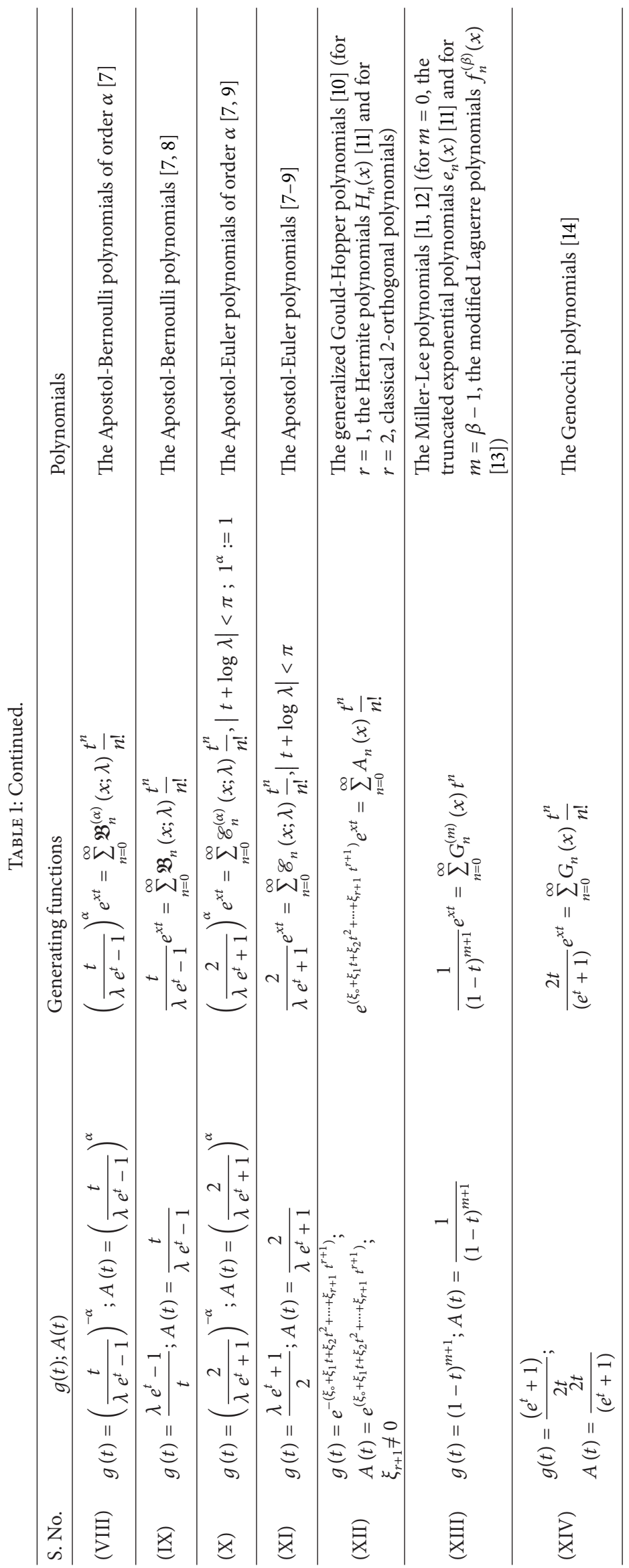


The operators $\widehat{M}$ and $\widehat{P}$ also satisfy the commutation relation

$$
[\widehat{P}, \widehat{M}]=\widehat{P} \widehat{M}-\widehat{M} \widehat{P}=\widehat{1}
$$

and thus display the Weyl group structure. If the considered polynomial set $\left\{p_{n}(x)\right\}_{n \in \mathbb{N}}$ is quasimonomial, its properties can easily be derived from those of the $\widehat{M}$ and $\widehat{P}$ operators. In fact,

(i) Combining the recurrences (6) and (7), we have

$$
\widehat{M} \widehat{P}\left\{p_{n}(x)\right\}=n p_{n}(x)
$$

which can be interpreted as the differential equation satisfied by $p_{n}(x)$, if $\widehat{M}$ and $\widehat{P}$ have a differential realization.

(ii) Assuming here and in the sequel $p_{0}(x)=1$, then $p_{n}(x)$ can be explicitly constructed as

$$
p_{n}(x)=\widehat{M}^{n}\left\{p_{0}(x)\right\}=\widehat{M}^{n}\{1\}
$$

which yields the series definition for $p_{n}(x)$.

(iii) Identity (10) implies that the exponential generating function of $p_{n}(x)$ can be given in the form

$$
\exp (t \widehat{M})\{1\}=\sum_{n=0}^{\infty} p_{n}(x) \frac{t^{n}}{n !} \quad(|t|<\infty)
$$

We note that the Appell polynomials $A_{n}(x)$ are quasimonomial with respect to the following multiplicative and derivative operators:

$$
\widehat{M}_{A}=x+\frac{A^{\prime}\left(D_{x}\right)}{A\left(D_{x}\right)}
$$

or, equivalently,

$$
\begin{gathered}
\widehat{M}_{A}=x-\frac{g^{\prime}\left(D_{x}\right)}{g\left(D_{\mathrm{x}}\right)}, \\
\widehat{P}_{A}=D_{x},
\end{gathered}
$$

respectively.

The special polynomials of two variables are useful from the point of view of applications in physics. Also, these polynomials allow the derivation of a number of useful identities in a fairly straightforward way and help in introducing new families of special polynomials. For example, Bretti et al. [17] introduced general classes of two variables Appell polynomials by using properties of an iterated isomorphism, related to the Laguerre-type exponentials.

We consider the 2-variable general polynomial (2VgP) family $p_{n}(x, y)$ defined by the generating function

$$
e^{x t} \phi(y, t)=\sum_{n=0}^{\infty} p_{n}(x, y) \frac{t^{n}}{n !} \quad\left(p_{0}(x, y)=1\right),
$$

where $\phi(y, t)$ has (at least the formal) series expansion

$$
\phi(y, t)=\sum_{n=0}^{\infty} \phi_{n}(y) \frac{t^{n}}{n !} \quad\left(\phi_{0}(y) \neq 0\right) .
$$

We recall that the 2-variable Hermite Kampé de Fériet polynomials (2VHKdFP) $H_{n}(x, y)$ [18], the Gould-Hopper polynomials (GHP) $H_{n}^{(m)}(x, y)$ [19], and the Hermite-Appell polynomials (HAP) ${ }_{H} A_{n}(x, y)$ [20] are defined by the generating functions

$$
\begin{gathered}
e^{x t+y t^{2}}=\sum_{n=0}^{\infty} H_{n}(x, y) \frac{t^{n}}{n !}, \\
e^{x t+y t^{m}}=\sum_{n=0}^{\infty} H_{n}^{(m)}(x, y) \frac{t^{n}}{n !}, \\
A(t) e^{x t+y t^{2}}=\sum_{n=0}^{\infty} H_{n}(x, y) \frac{t^{n}}{n !},
\end{gathered}
$$

respectively. Thus, in view of generating functions (14), (16), (17), and (18), we note that the 2VHKdFP $H_{n}(x, y)$, the GHP $H_{n}^{(m)}(x, y)$, and the HAP ${ }_{H} A_{n}(x, y)$ belong to $2 \mathrm{VgP}$ family.

In this paper, operational methods are used to introduce certain new families of special polynomials related to the Appell polynomials. In Section 2, some results for the 2variable general polynomials $(2 \mathrm{VgP}) p_{n}(x, y)$ are derived. Further, the 2-variable general-Appell polynomials (2VgAP) ${ }_{p} A_{n}(x, y)$ are introduced and framed within the context of monomiality principle. In Section 3, the Gould-HopperAppell polynomials (GHAP) ${ }_{H} A_{n}^{(m)}(x, y)$ are considered, and their properties are established. Some members belonging to the Gould-Hopper-Appell polynomial family are given.

\section{2-Variable General-Appell Polynomials}

In order to introduce the 2-variable general-Appell polynomials (2VgAP), we need to establish certain results for the $2 \mathrm{VgP} p_{n}(x, y)$. Therefore, first we prove the following results for the $2 \operatorname{VgP} p_{n}(x, y)$.

Lemma 1. The $2 \operatorname{VgP} p_{n}(x, y)$ defined by generating function (14), where $\phi(y, t)$ is given by (15), are quasimonomial under the action of the following multiplicative and derivative operators:

$$
\begin{gathered}
\widehat{M}_{p}=x+\frac{\phi^{\prime}\left(y, D_{x}\right)}{\phi\left(y, D_{x}\right)} \quad\left(\phi^{\prime}(x, t):=\frac{\partial}{\partial t} \phi(x, t)\right), \\
\widehat{P}_{p}=D_{x},
\end{gathered}
$$

respectively.

Proof. Differentiating (14) partially with respect to $t$, we have

$$
\left(x+\frac{\phi^{\prime}(y, t)}{\phi(y, t)}\right)\left\{e^{x t} \phi(y, t)\right\}=\sum_{n=0}^{\infty} p_{n+1}(x, y) \frac{t^{n}}{n !} .
$$




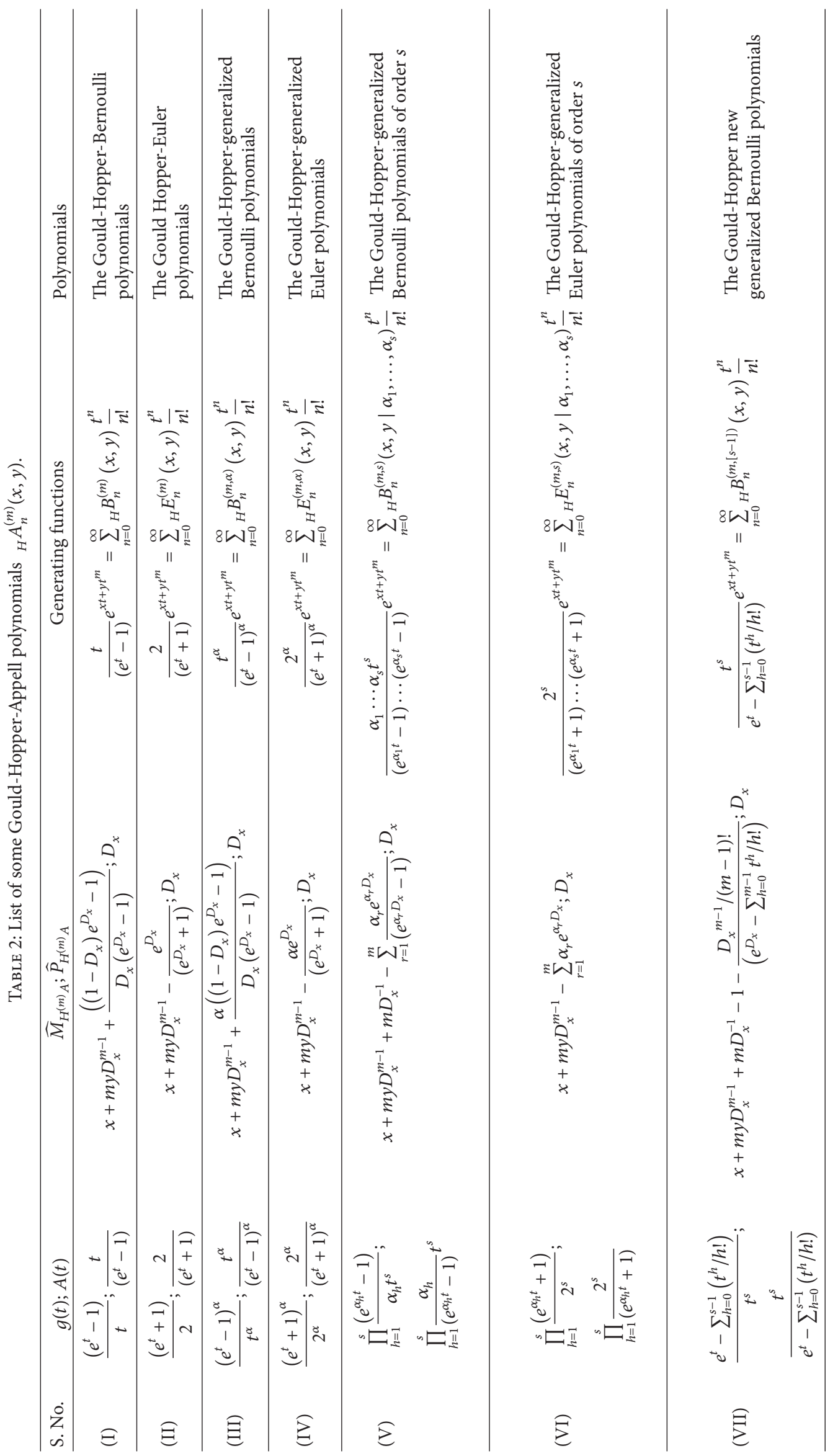




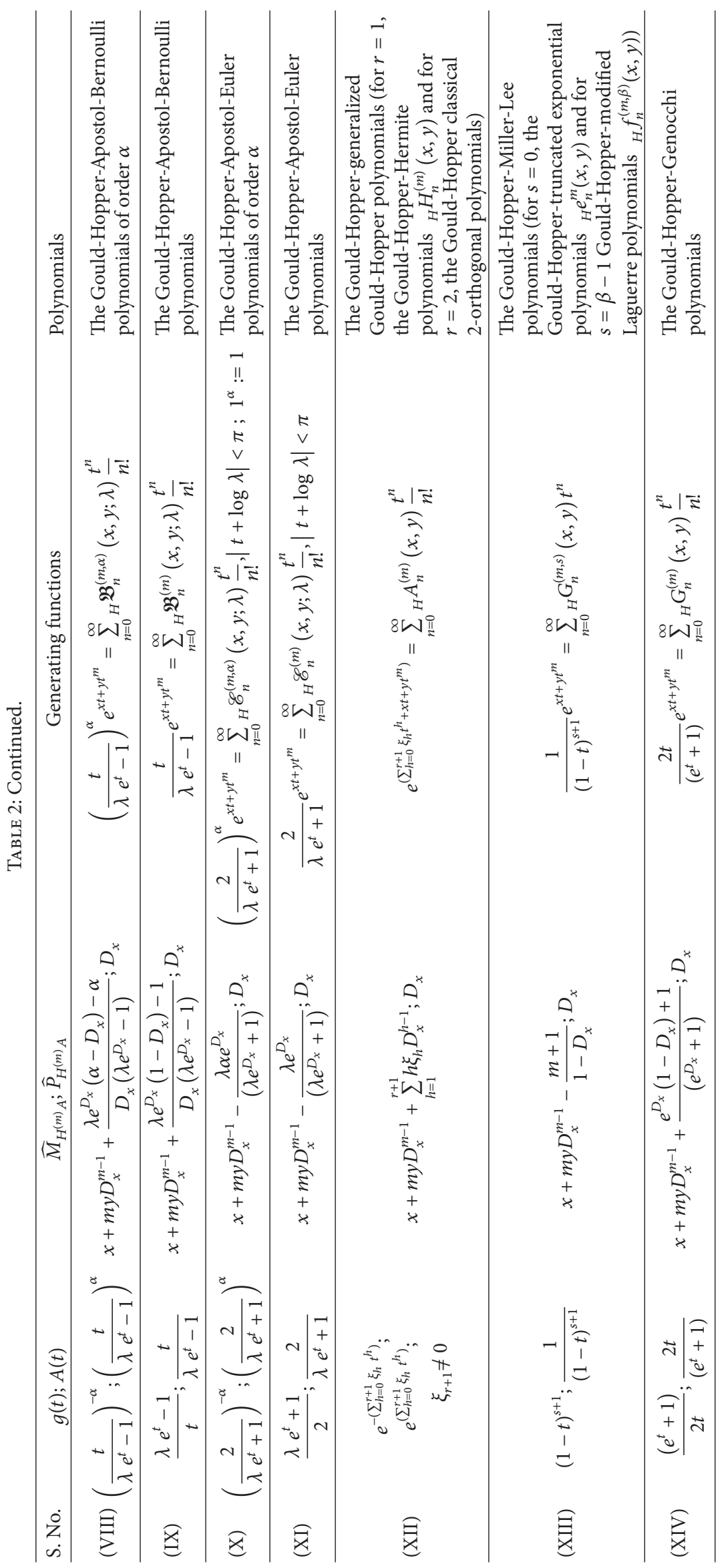


If $\phi(y, t)$ is an invertible series and $\phi^{\prime}(y, t) / \phi(y, t)$ has Taylor's series expansion in powers of $t$, then in view of the identity

$$
D_{x}\left\{e^{x t} \phi(y, t)\right\}=t\left(e^{x t} \phi(y, t)\right)
$$

we can write

$$
\frac{\phi^{\prime}\left(y, D_{x}\right)}{\phi\left(y, D_{x}\right)}\left\{e^{x t} \phi(y, t)\right\}=\frac{\phi^{\prime}(y, t)}{\phi(y, t)}\left(e^{x t} \phi(y, t)\right) .
$$

Now, using (23) in the l.h.s. of (21), we find

$$
\left(x+\frac{\phi^{\prime}\left(y, D_{x}\right)}{\phi\left(y, D_{x}\right)}\right)\left\{e^{x t} \phi(y, t)\right\}=\sum_{n=0}^{\infty} p_{n+1}(x, y) \frac{t^{n}}{n !} .
$$

Making use of generating function (14) in the l.h.s. of the above equation, we have

$$
\left(x+\frac{\phi^{\prime}\left(y, D_{x}\right)}{\phi\left(y, D_{x}\right)}\right)\left\{\sum_{n=0}^{\infty} p_{n}(x, y) \frac{t^{n}}{n !}\right\}=\sum_{n=0}^{\infty} p_{n+1}(x, y) \frac{t^{n}}{n !}
$$

which, on equating the coefficients of like powers of $t$ in both sides, gives

$$
\left(x+\frac{\phi^{\prime}\left(y, D_{x}\right)}{\phi\left(y, D_{x}\right)}\right)\left\{p_{n}(x, y)\right\}=p_{n+1}(x, y) .
$$

Thus, in view of monomiality principle equation (6), the above equation yields assertion (19) of Lemma 1. Again, using identity (22) in (14), we have

$$
D_{x}\left\{\sum_{n=0}^{\infty} p_{n}(x, y) \frac{t^{n}}{n !}\right\}=\sum_{n=1}^{\infty} p_{n-1}(x, y) \frac{t^{n}}{(n-1) !} .
$$

Equating the coefficients of like powers of $t$ in both sides of (27), we find

$$
D_{x}\left\{p_{n}(x, y)\right\}=n p_{n-1}(x, y) \quad(n \geq 1)
$$

which in view of monomiality principle equation (7) yields assertion (20) of Lemma 1.

Remark 2. The operators given by (19) and (20) satisfy commutation relation (8). Also, using expressions (19) and (20) in (9), we get the following differential equation satisfied by $2 \mathrm{VgP} p_{n}(x, y)$ :

$$
\left(x D_{x}+\frac{\phi^{\prime}\left(y, D_{x}\right)}{\phi\left(y, D_{x}\right)} D_{x}-n\right) p_{n}(x, y)=0 .
$$

Remark 3. Since $p_{0}(x, y)=1$, therefore, in view of monomiality principle equation (10), we have

$$
p_{n}(x, y)=\left(x+\frac{\phi^{\prime}\left(y, D_{x}\right)}{\phi\left(y, D_{x}\right)}\right)^{n}\{1\} \quad\left(p_{0}(x, y)=1\right) .
$$

Also, in view of (11), (14), and (19), we have

$$
\exp \left(\widehat{M}_{p} t\right)\{1\}=e^{x t} \phi(y, t)=\sum_{n=0}^{\infty} p_{n}(x, y) \frac{t^{n}}{n !} .
$$

Now, we proceed to introduce the 2-variable generalAppell polynomials (2VgAP). In order to derive the generating functions for the $2 \mathrm{VgAP}$, we take the $2 \mathrm{VgP} p_{n}(x, y)$ as the base in the Appell polynomials generating function (1). Thus, replacing $x$ by the multiplicative operator $\widehat{M}_{p}$ of the $2 \operatorname{VgP} p_{n}(x, y)$ in the l.h.s. of (1) and denoting the resultant 2VgAP by ${ }_{p} A_{n}(x, y)$, we have

$$
A(t) e^{\left(\widehat{M}_{p} t\right)}=\sum_{n=0}^{\infty} A_{n}(x, y) \frac{t^{n}}{n !} .
$$

Now, using (31) in the exponential term in the 1.h.s. of (32), we get the generating function for ${ }_{p} A_{n}(x, y)$ as

$$
A(t) e^{x t} \phi(y, t)=\sum_{n=0}^{\infty}{ }_{p} A_{n}(x, y) \frac{t^{n}}{n !} .
$$

In view of (5), generating function (33) can be expressed equivalently as

$$
\frac{1}{g(t)} e^{x t} \phi(y, t)=\sum_{n=0}^{\infty}{ }_{p} A_{n}(x, y) \frac{t^{n}}{n !} .
$$

Now, we frame the $2 \operatorname{VgAP}{ }_{p} A_{n}(x, y)$ within the context of monomiality principle formalism. We prove the following results.

Theorem 4. The $2 \operatorname{VgAP}{ }_{p} A_{n}(x, y)$ are quasimonomial with respect to the following multiplicative and derivative operators:

$$
\widehat{M}_{p A}=x+\frac{\phi^{\prime}\left(y, D_{x}\right)}{\phi\left(y, D_{x}\right)}+\frac{A^{\prime}\left(D_{x}\right)}{A\left(D_{x}\right)}
$$

or, equivalently,

$$
\begin{gathered}
\widehat{M}_{p A}=x+\frac{\phi^{\prime}\left(y, D_{x}\right)}{\phi\left(y, D_{x}\right)}-\frac{g^{\prime}\left(D_{x}\right)}{g\left(D_{x}\right)}, \\
\widehat{P}_{p A}=D_{x},
\end{gathered}
$$

respectively.

Proof. Differentiating (33) partially with respect to $t$, we find

$$
\begin{aligned}
(x & \left.+\frac{\phi^{\prime}(y, t)}{\phi(y, t)}+\frac{A^{\prime}(t)}{A(t)}\right) A(t) e^{x t} \phi(y, t) \\
= & \sum_{n=0}^{\infty}{ }_{p} A_{n+1}(x, y) \frac{t^{n}}{n !} .
\end{aligned}
$$

Since $A(t)$ and $\phi(y, t)$ are invertible series of $t$, therefore, $A^{\prime}(t) / A(t)$ and $\phi^{\prime}(y, t) / \phi(y, t)$ possess power series expansions of $t$. Thus, in view of identity (22), we have

$$
\begin{aligned}
(x+ & \left.\frac{\phi^{\prime}\left(y, D_{x}\right)}{\phi\left(y, D_{x}\right)}+\frac{A^{\prime}\left(D_{x}\right)}{A\left(D_{x}\right)}\right)\left\{A(t) e^{x t} \phi(y, t)\right\} \\
= & \sum_{n=0}^{\infty}{ }_{p} A_{n+1}(x, y) \frac{t^{n}}{n !}
\end{aligned}
$$


which, on using generating function (33), becomes

$$
\begin{aligned}
(x & \left.+\frac{\phi^{\prime}\left(y, D_{x}\right)}{\phi\left(y, D_{x}\right)}+\frac{A^{\prime}\left(D_{x}\right)}{A\left(D_{x}\right)}\right)\left\{\sum_{n=0}^{\infty}{ }_{p} A_{n}(x, y) \frac{t^{n}}{n !}\right\} \\
= & \sum_{n=0}^{\infty}{ }_{p} A_{n+1}(x, y) \frac{t^{n}}{n !}
\end{aligned}
$$

or, equivalently,

$$
\begin{aligned}
\sum_{n=0}^{\infty} & \left(x+\frac{\phi^{\prime}\left(y, D_{x}\right)}{\phi\left(y, D_{x}\right)}+\frac{A^{\prime}\left(D_{x}\right)}{A\left(D_{x}\right)}\right)\left\{{ }_{p} A_{n}(x, y)\right\} \frac{t^{n}}{n !} \\
& =\sum_{n=0}^{\infty}{ }_{p} A_{n+1}(x, y) \frac{t^{n}}{n !}
\end{aligned}
$$

Now, equating the coefficients of like powers of $t$ in the above equation, we find

$$
\left(x+\frac{\phi^{\prime}\left(y, D_{x}\right)}{\phi\left(y, D_{x}\right)}+\frac{A^{\prime}\left(D_{x}\right)}{A\left(D_{x}\right)}\right)\left\{{ }_{p} A_{n}(x, y)\right\}={ }_{p} A_{n+1}(x, y)
$$

which in view of (6) yields assertion (35a) of Theorem 4. Also, in view of relation (5), assertion (35a) can be expressed equivalently as (35b).

Again, in view of identity (22), we have

$$
D_{x}\left\{A(t) e^{x t} \phi(y, t)\right\}=t A(t) e^{x t} \phi(y, t)
$$

which on using generating function (33) becomes

$$
D_{x}\left\{\sum_{n=0}^{\infty}{ }_{p} A_{n}(x, y) \frac{t^{n}}{n !}\right\}=\sum_{n=1}^{\infty}{ }_{p} A_{n-1}(x, y) \frac{t^{n}}{(n-1) !} .
$$

Equating the coefficients of like powers of $t$ in the above equation, we find

$$
D_{x}\left\{{ }_{p} A_{n}(x, y)\right\}=n_{p} A_{n-1}(x, y) \quad(n \geq 1)
$$

which, in view of (7), yields assertion (36) of Theorem 4.

Theorem 5. The $2 \operatorname{VgAP}{ }_{p} A_{n}(x, y)$ satisfy the following differential equations

$$
\left(x D_{x}+\frac{\phi^{\prime}\left(y, D_{x}\right)}{\phi\left(y, D_{x}\right)} D_{x}+\frac{A^{\prime}\left(D_{x}\right)}{A\left(D_{x}\right)} D_{x}-n\right){ }_{p} A_{n}(x, y)=0
$$

or, equivalently,

$$
\left(x D_{x}+\frac{\phi^{\prime}\left(y, D_{x}\right)}{\phi\left(y, D_{x}\right)} D_{x}-\frac{g^{\prime}\left(D_{x}\right)}{g\left(D_{x}\right)} D_{x}-n\right){ }_{p} A_{n}(x, y)=0 .
$$

Proof. Using (35a) and (36) in (9), we get assertion (45a). Further, using (35b) and (36) in (9), we get assertion (45b).

Note 1. With the help of the results derived above and by taking $A(t)$ (or $g(t)$ ) of the Appell polynomials listed in Table 1, we can derive the generating function and other results for the members belonging to $2 \mathrm{VgAP}$ family.

\section{Examples}

We consider examples of certain members belonging to the 2VgAP family.

Taking $\phi(y, t)=e^{y t^{m}}$ (that is, when the $2 \operatorname{VgP} p_{n}(x, y)$ reduces to the GHP $\left.H_{n}^{(m)}(x, y)\right)$ in generating function (33), we find that the Gould-Hopper-Appell polynomials (GHAP) ${ }_{H} A_{n}^{(m)}(x, y)$ are defined by the following generating function:

$$
A(t) e^{\left(x t+y t^{m}\right)}=\sum_{n=0}^{\infty}{ }_{H} A_{n}^{(m)}(x, y) \frac{t^{n}}{n !}
$$

or, equivalently,

$$
\frac{1}{g(t)} e^{\left(x t+y t^{m}\right)}=\sum_{n=0}^{\infty} H_{n}^{(m)}(x, y) \frac{t^{n}}{n !} .
$$

Using (1) in (46) (or (3) in (47)), we get the following series definition for ${ }_{H} A_{n}^{(m)}(x, y)$ in terms of the Appell polynomials $A_{n}(x)$ :

$$
{ }_{H} A_{n}^{(m)}(x, y)=n ! \sum_{r=0}^{[n / m]} \frac{A_{n-m r}(x) y^{r}}{(n-m r) ! r !} .
$$

In view of (35a), (35b), and (36), we note that the GHAP ${ }_{H} A_{n}^{(m)}(x, y)$ are quasimonomial under the action of the following multiplicative and derivative operators:

$$
\widehat{M}_{H^{(m)} A}=x+m y D_{x}^{m-1}+\frac{A^{\prime}\left(D_{x}\right)}{A\left(D_{x}\right)}
$$

or, equivalently,

$$
\widehat{M}_{H^{(m)} A}=x+m y D_{x}^{m-1}-\frac{g^{\prime}\left(D_{x}\right)}{g\left(D_{x}\right)},
$$

$$
\widehat{P}_{H^{(m)} A}=D_{x},
$$

respectively. Also, in view of (45a) and (45b), we find that the GHAP ${ }_{H} A_{n}^{(m)}(x, y)$ satisfy the following differential equation:

$$
\left(x D_{x}+m y D_{x}^{m}+\frac{A^{\prime}\left(D_{x}\right)}{A\left(D_{x}\right)} D_{x}-n\right){ }_{H} A_{n}^{(m)}(x, y)=0
$$

or, equivalently,

$$
\left(x D_{x}+m y D_{x}^{m}-\frac{g^{\prime}\left(D_{x}\right)}{g\left(D_{x}\right)} D_{x}-n\right){ }_{H} A_{n}^{(m)}(x, y)=0 .
$$

Remark 6. In view of (16) and (17), we note that, for $m=$ 2, the GHAP ${ }_{H} A_{n}^{(m)}(x, y)$ reduce to the Hermite-Appell polynomials (HAP) ${ }_{H} A_{n}(x, y)$. Therefore, taking $m=2$ in (46), (47), (48), (49a), (49b), (50), (51a), and (51b), we get the corresponding results for the HAP ${ }_{H} A_{n}(x, y)$. 
Remark 7. In view of (16), we note that the 2VHKdFP $H_{n}(x, y)$ are related to the classical Hermite polynomials $H_{n}(x)[11]$ or $\mathrm{He}_{n}(x)$ as

$$
\begin{gathered}
H_{n}(2 x,-1)=H_{n}(x), \\
H_{n}\left(x,-\frac{1}{2}\right)=H e_{n}(x) .
\end{gathered}
$$

Therefore, taking $y=-1$ and replacing $x$ by $2 x$ (or taking $y=-1 / 2)$ in (46)-(51b), we get the corresponding results for the classical Hermite-Appell polynomials ${ }_{H} A_{n}(x)$ (or $\left.{ }_{H e} A_{n}(x)\right)$.

There are several members belonging to $2 \mathrm{VgP}$ family. Thus the results for the corresponding $2 \mathrm{VgAP}$ can be obtained by taking other examples. We present the list of some members belonging to the GHAP family in Table 2 .

Note 2. Since, for $m=2$, the GHAP ${ }_{H} A_{n}^{m}(x, y)$ reduce to the HAP ${ }_{H} A_{n}(x, y)$ therefore, for $m=2$, Table 2 gives the list of the corresponding $\mathrm{HAP}{ }_{H} A_{n}(x, y)$.

The results established in this paper are general and include new families of special polynomials, consequently introducing many new special polynomials.

\section{Appendix}

New classes of Bernoulli numbers and polynomials are introduced, which are used to evaluate partial sums involving other special polynomials, see, for example, [21, 22]. Here, we consider the Gould-Hopper-Bernoulli polynomials (GHBP), Gould-Hopper-Euler polynomials (GHEP), Hermite-Bernoulli polynomials (HBP), Hermite-Euler polynomials (HEP), classical Hermite-Bernoulli polynomials (cHBP), and classical Hermite-Euler polynomials (cHEP). We give the surface plots of these polynomials for suitable values of the parameters and indices. Also, we give the graphs of the corresponding single-variable polynomials.

The GHBP ${ }_{H} B_{n}^{m}(x, y)$, GHEP ${ }_{H} E_{n}^{m}(x, y)$, HBP ${ }_{H} B_{n}(x, y)$, and $\operatorname{HEP}{ }_{H} E_{n}(x, y)$ are defined by the following series:

$$
\begin{aligned}
{ }_{H} B_{n}^{(m)}(x, y) & =n ! \sum_{r=0}^{[n / m]} \frac{B_{n-m r}(x) y^{r}}{(n-m r) ! r !} \\
{ }_{H} E_{n}^{(m)}(x, y) & =n ! \sum_{r=0}^{[n / m]} \frac{E_{n-m r}(x) y^{r}}{(n-m r) ! r !} \\
{ }_{H} B_{n}(x, y) & =n ! \sum_{r=0}^{[n / 2]} \frac{B_{n-2 r}(x) y^{r}}{(n-2 r) ! r !} \\
{ }_{H} E_{n}(x, y) & =n ! \sum_{r=0}^{[n / 2]} \frac{E_{n-2 r}(x) y^{r}}{(n-2 r) ! r !}
\end{aligned}
$$

respectively. Taking $y=-1 / 2$ in (A.3) and (A.4), we get the series definitions for the $\mathrm{CHBP}{ }_{\mathrm{He}} B_{n}(x)$ and $\mathrm{cHEP}{ }_{\mathrm{He}} E_{n}(x)$ as

$$
\begin{aligned}
& { }_{H} B_{n}(x)=n ! \sum_{r=0}^{[n / 2]}\left(\frac{-1}{2}\right)^{r} \frac{B_{n-2 r}(x)}{(n-2 r) ! r !}, \\
& { }_{H} E_{n}(x)=n ! \sum_{r=0}^{[n / 2]}\left(\frac{-1}{2}\right)^{r} \frac{E_{n-2 r}(x)}{(n-2 r) ! r !},
\end{aligned}
$$

respectively.

To draw the surface plots of these polynomials, we use the values of the Bernoulli polynomials $B_{n}(x)$ and the Euler polynomials $E_{n}(x)$ for $n=0,1,2,3,4$, and 5 . We give the list of the first few Bernoulli and the Euler polynomials in Table 3.

Now, we consider the GHBP ${ }_{H} B_{n}^{m}(x, y)$, GHEP ${ }_{H} E_{n}^{m}(x, y), \quad \mathrm{HBP} \quad{ }_{H} B_{n}(x, y), \quad \mathrm{HEP}{ }_{H} E_{n}(x, y), \quad$ cHBP ${ }_{H e} B_{n}(x)$, and $\mathrm{cHEP}{ }_{H e} E_{n}(x)$, for $m=3$ and $n=5$, so that we have

$$
\begin{gathered}
{ }_{H} B_{5}^{(3)}(x, y)=B_{5}(x)+60 B_{2}(x) y, \\
{ }_{H} E_{5}^{(3)}(x, y)=E_{5}(x)+60 E_{2}(x) y, \\
{ }_{H} B_{5}(x, y)=B_{5}(x)+20 B_{3}(x) y+60 B_{1}(x) y^{2}, \\
{ }_{H} E_{5}(x, y)=E_{5}(x)+20 E_{3}(x) y+60 E_{1}(x) y^{2}, \\
{ }_{H e} B_{5}(x)=B_{5}(x)-10 B_{3}(x)+15 B_{1}(x), \\
{ }_{H e} E_{5}(x)=E_{5}(x)-10 E_{3}(x)+15 E_{1}(x),
\end{gathered}
$$

respectively. Using the particular values of $B_{n}(x)$ and $E_{n}(x)$ given in Table 3, we find

$$
\begin{aligned}
& { }_{H} B_{5}^{(3)}(x, y)=x^{5}-\frac{5}{2} x^{4}+\frac{5}{3} x^{3}+60 x^{2} y-60 x y-\frac{1}{6} x+\frac{1}{10} y \\
& { }_{H} E_{5}^{(3)}(x, y)=x^{5}-\frac{5}{2} x^{4}+60 x^{2} y+\frac{5}{3} x^{2}-60 x y-\frac{1}{2}+\frac{1}{10} y
\end{aligned}
$$

$$
\begin{aligned}
{ }_{H} B_{5}(x, y)= & x^{5}-\frac{5}{2} x^{4}+20 x^{3} y+\frac{5}{3} x^{3}+60 x y^{2} \\
& -60 x^{2} y+10 x y-30 y^{2}-\frac{1}{6} x, \\
{ }_{H} E_{5}(x, y)= & x^{5}-\frac{5}{2} x^{4}+20 x^{3} y+60 x y^{2} \\
& -30 x^{2} y+\frac{5}{3} x^{2}-30 y^{2}+\frac{10}{3} y-\frac{1}{2}, \\
H_{e} B_{5}(x)=x^{5}- & \frac{5}{2} x^{4}-\frac{25}{3} x^{3}+15 x^{2}+\frac{59}{6} x-\frac{15}{2}, \\
H_{e} E_{5}(x)=x^{5}- & \frac{5}{2} x^{4}-10 x^{3}+\frac{50}{3} x^{2}+15 x-\frac{29}{3},
\end{aligned}
$$

respectively. 


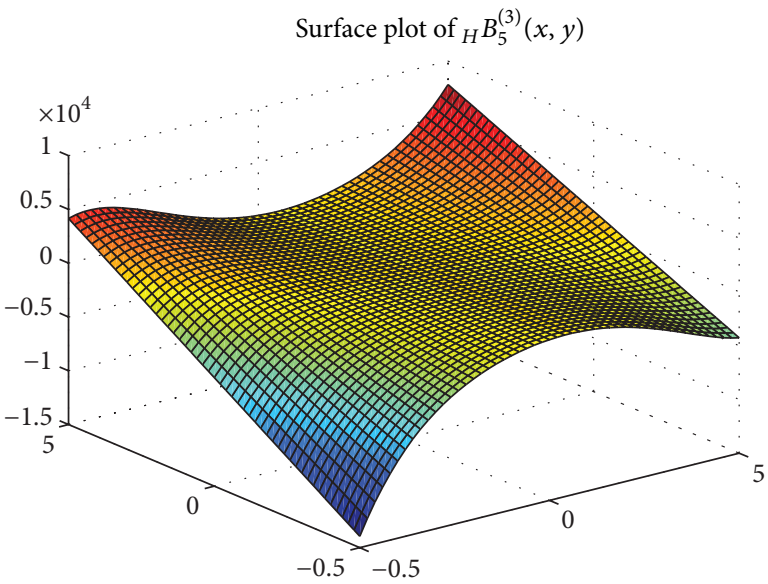

(a)

Surface plot of ${ }_{H} B_{5}(x, y)$

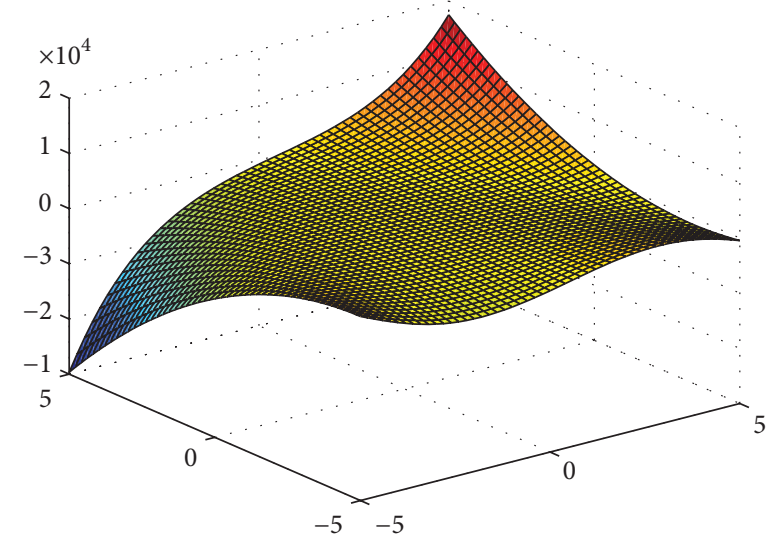

(c)

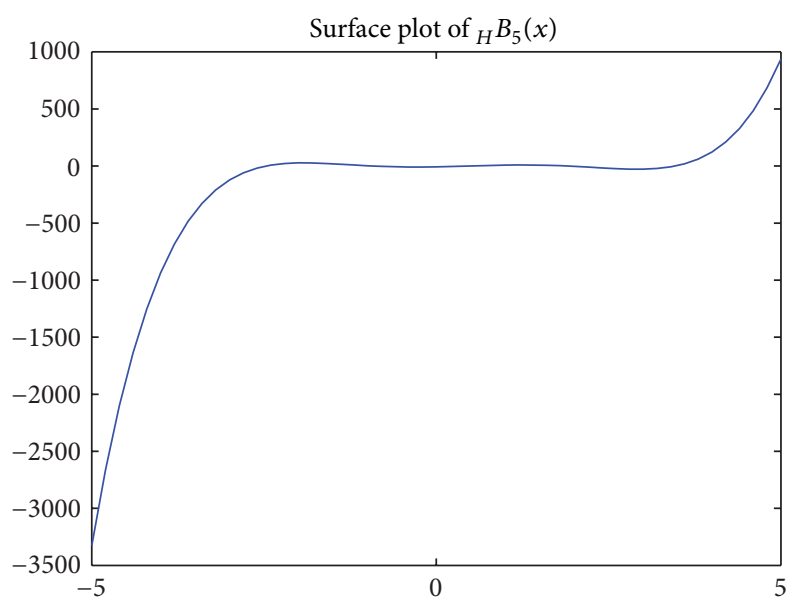

(e)

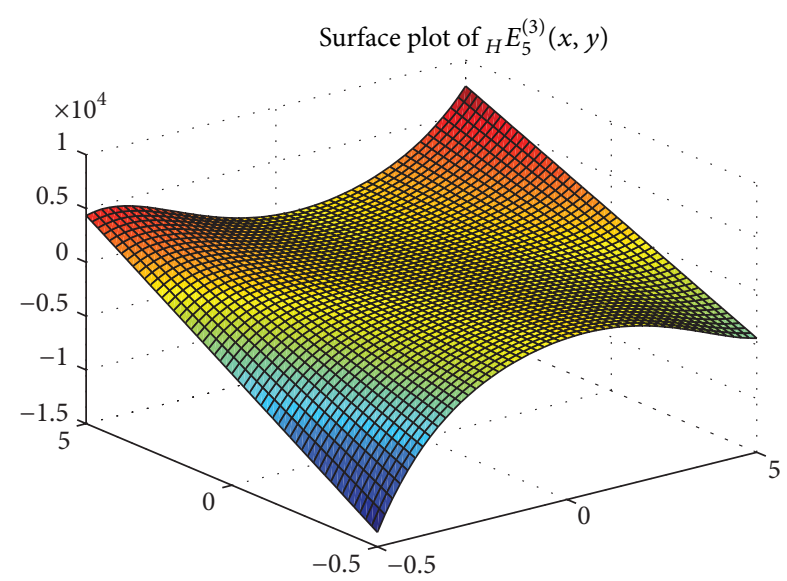

(b)

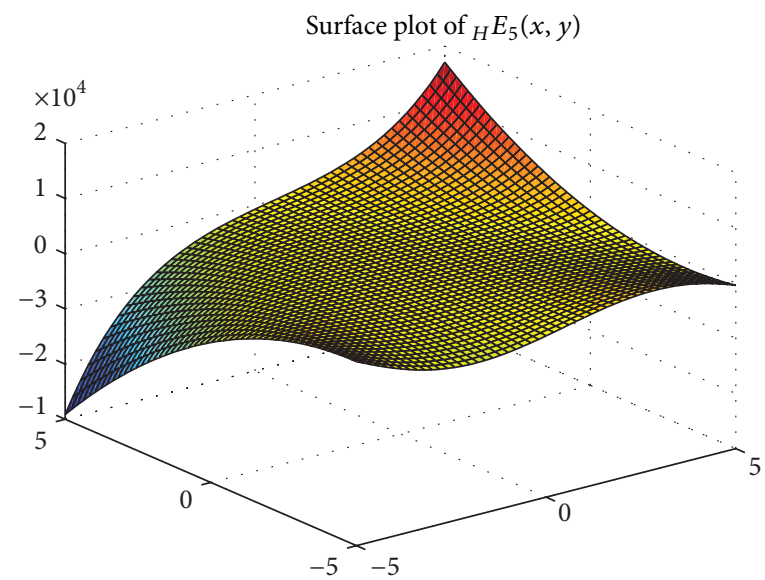

(d)

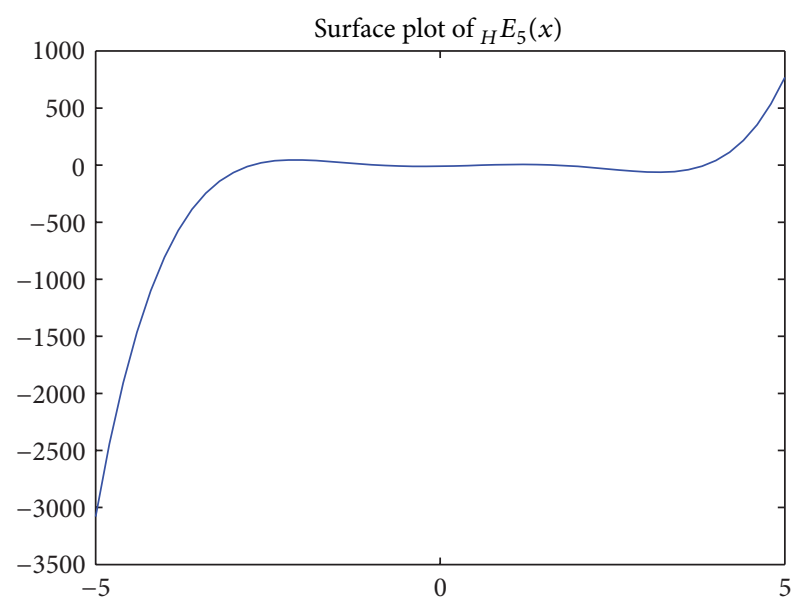

(f)

Figure 1

TABLE 3: List of the first few Bernoulli and the Euler polynomials.

\begin{tabular}{llccccc}
\hline$n$ & 0 & 1 & 2 & 3 & 4 & 5 \\
\hline$B_{n}(x)$ & 1 & $x-\frac{1}{2}$ & $x^{2}-x+\frac{1}{6}$ & $x^{3}-\frac{3}{2} x^{2}+\frac{x}{2}$ & $x^{4}-2 x^{3}+x^{2}-\frac{1}{30}$ & $x^{5}-\frac{5}{2} x^{4}+\frac{5}{3} x^{3}-\frac{x}{6}$ \\
$E_{n}(x)$ & 1 & $x-\frac{1}{2}$ & $x^{2}-x$ & $x^{3}-\frac{3}{2} x^{2}+\frac{1}{6}$ & $x^{4}-2 x^{3}+\frac{2}{3} x$ & $x^{5}-\frac{5}{2} x^{4}+\frac{5}{3} x^{2}-\frac{1}{2}$ \\
\hline
\end{tabular}


In view of equations (A.13)-(A.18), we get Figure 1.

\section{Acknowledgments}

The authors are thankful to the anonymous referee for useful suggestions towards the improvement of the paper. This work has been done under the Senior Research Fellowship (Office Memo no. Acad/D-1562/MR) sanctioned to the second author by the University Grants Commission, Government of India, New Delhi.

\section{References}

[1] P. Appell, "Sur une classe de polynômes," Annales Scientifiques de l'École Normale Supérieure, vol. 9, pp. 119-144, 1880.

[2] S. Roman, The Umbral Calculus, vol. 111 of Pure and Applied Mathematics, Academic Press, New York, NY, USA, 1984.

[3] E. D. Rainville, Special Functions, Macmillan, New York, NY, USA, 1960, reprinted by Chelsea, Bronx, NY, USA, 1971.

[4] A. Erdélyi, W. Magnus, F. Oberhettinger, and F. G. Tricomi, Higher Transcendental Functions. Vol. III, McGraw-Hill, New York, NY, USA, 1955.

[5] A. Erdélyi, W. Magnus, F. Oberhettinger, and F. G. Tricomi, Higher Transcendental Functions. Vol. II, McGraw-Hill, New York, NY, USA, 1953.

[6] G. Bretti, P. Natalini, and P. E. Ricci, "Generalizations of the Bernoulli and Appell polynomials," Abstract and Applied Analysis, vol. 2004, no. 7, pp. 613-623, 2004.

[7] Q.-M. Luo and H. M. Srivastava, "Some generalizations of the Apostol-Bernoulli and Apostol-Euler polynomials," Journal of Mathematical Analysis and Applications, vol. 308, no. 1, pp. 290302, 2005.

[8] T. M. Apostol, "On the Lerch zeta function," Pacific Journal of Mathematics, vol. 1, pp. 161-167, 1951.

[9] Q.-M. Luo, "Apostol-Euler polynomials of higher order and Gaussian hypergeometric functions," Taiwanese Journal of Mathematics, vol. 10, no. 4, pp. 917-925, 2006.

[10] K. Douak, "The relation of the $d$-orthogonal polynomials to the Appell polynomials," Journal of Computational and Applied Mathematics, vol. 70, no. 2, pp. 279-295, 1996.

[11] L. C. Andrews, Special Functions for Engineers and Applied Mathematicians, Macmillan, New York, NY, USA, 1985.

[12] G. Dattoli, S. Lorenzutta, and D. Sacchetti, "Integral representations of new families of polynomials," Italian Journal of Pure and Applied Mathematics, no. 15, pp. 19-28, 2004.

[13] W. Magnus, F. Oberhettinger, and R. P. Soni, Formulas and Theorems for the Special Functions of Mathematical Physics, vol. 52 of Die Grundlehren der mathematischen Wissenschaften, Springer, New York, NY, USA, 3rd edition, 1966.

[14] G. Dattoli, M. Migliorati, and H. M. Srivastava, "Sheffer polynomials, monomiality principle, algebraic methods and the theory of classical polynomials," Mathematical and Computer Modelling, vol. 45, no. 9-10, pp. 1033-1041, 2007.

[15] J. F. Steffensen, "The poweroid, an extension of the mathematical notion of power," Acta Mathematica, vol. 73, pp. 333-366, 1941.

[16] G. Dattoli, "Hermite-Bessel and Laguerre-Bessel functions: a by-product of the monomiality principle," in Advanced Special Functions and Applications (Melfi, 1999), vol. 1 of Proc. Melfi Sch. Adv. Top. Math. Phys., pp. 147-164, Aracne, Rome, Italy, 2000.
[17] G. Bretti, C. Cesarano, and P. E. Ricci, "Laguerre-type exponentials and generalized Appell polynomials," Computers \& Mathematics with Applications, vol. 48, no. 5-6, pp. 833-839, 2004.

[18] P. Appell and J. Kampé de Fériet, Fonctions Hypergéométriques et Hypersphériques: Polynômes d' Hermite, Gauthier-Villars, Paris, France, 1926.

[19] H. W. Gould and A. T. Hopper, "Operational formulas connected with two generalizations of Hermite polynomials," Duke Mathematical Journal, vol. 29, pp. 51-63, 1962.

[20] S. Khan, G. Yasmin, R. Khan, and N. A. M. Hassan, "Hermitebased Appell polynomials: properties and applications," Journal of Mathematical Analysis and Applications, vol. 351, no. 2, pp. 756-764, 2009.

[21] G. Dattoli, C. Cesarano, and S. Lorenzutta, "Bernoulli numbers and polynomials from a more general point of view," Rendiconti di Matematica e delle sue Applicazioni, vol. 22, pp. 193-202, 2002.

[22] G. Dattoli, S. Lorenzutta, and C. Cesarano, "Finite sums and generalized forms of Bernoulli polynomials," Rendiconti di Matematica e delle sue Applicazioni, vol. 19, no. 3, pp. 385-391, 1999. 


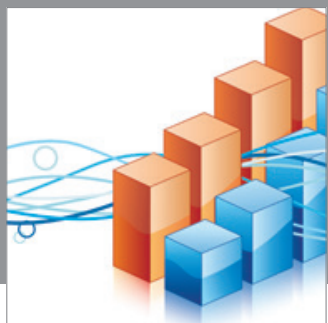

Advances in

Operations Research

mansans

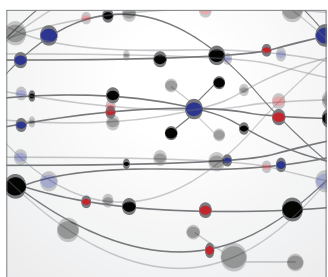

The Scientific World Journal
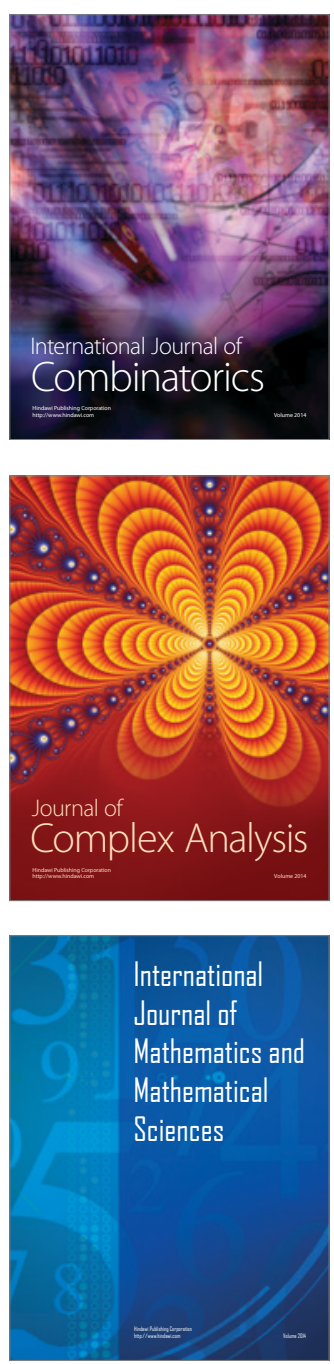
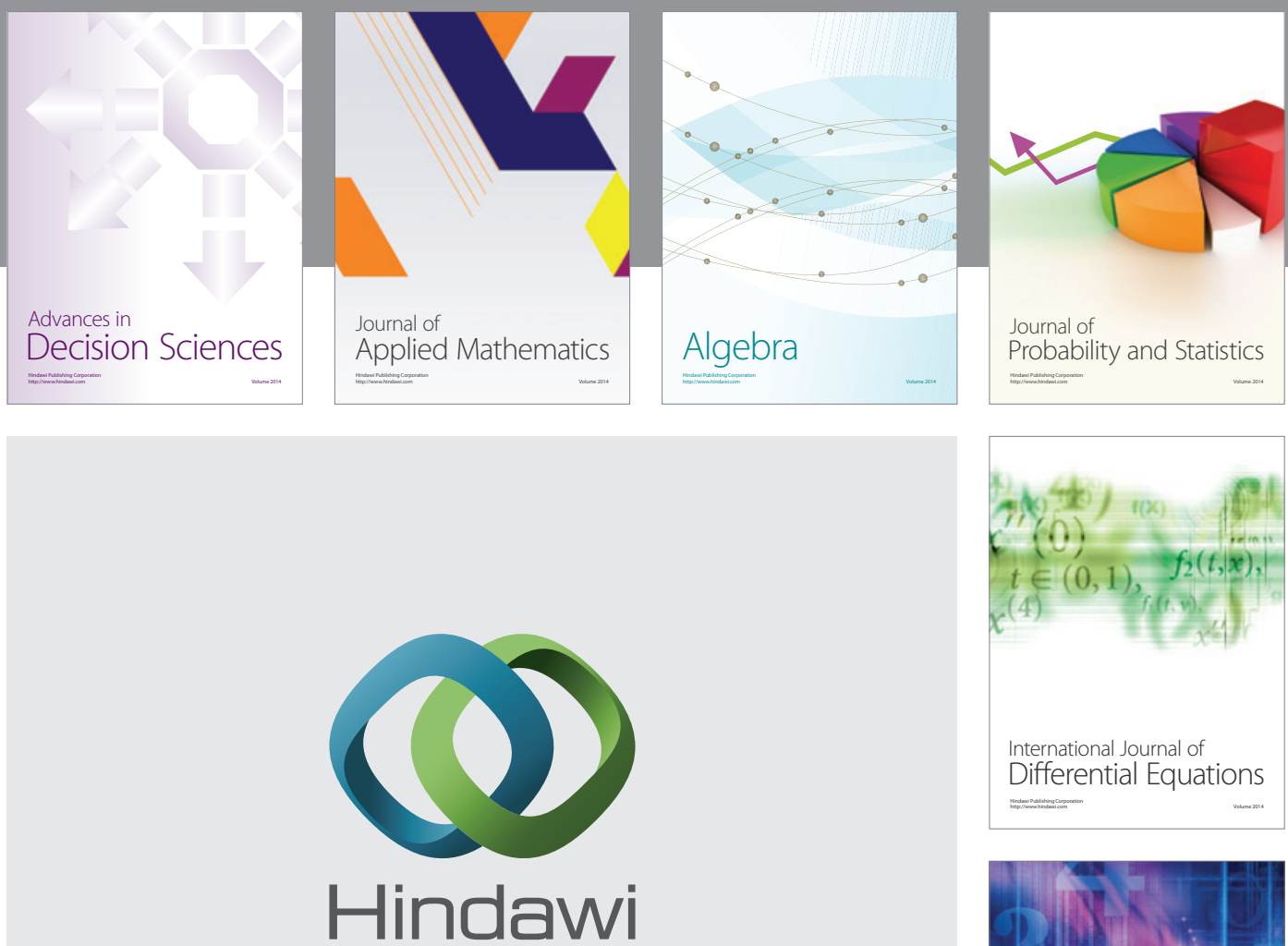

Submit your manuscripts at http://www.hindawi.com
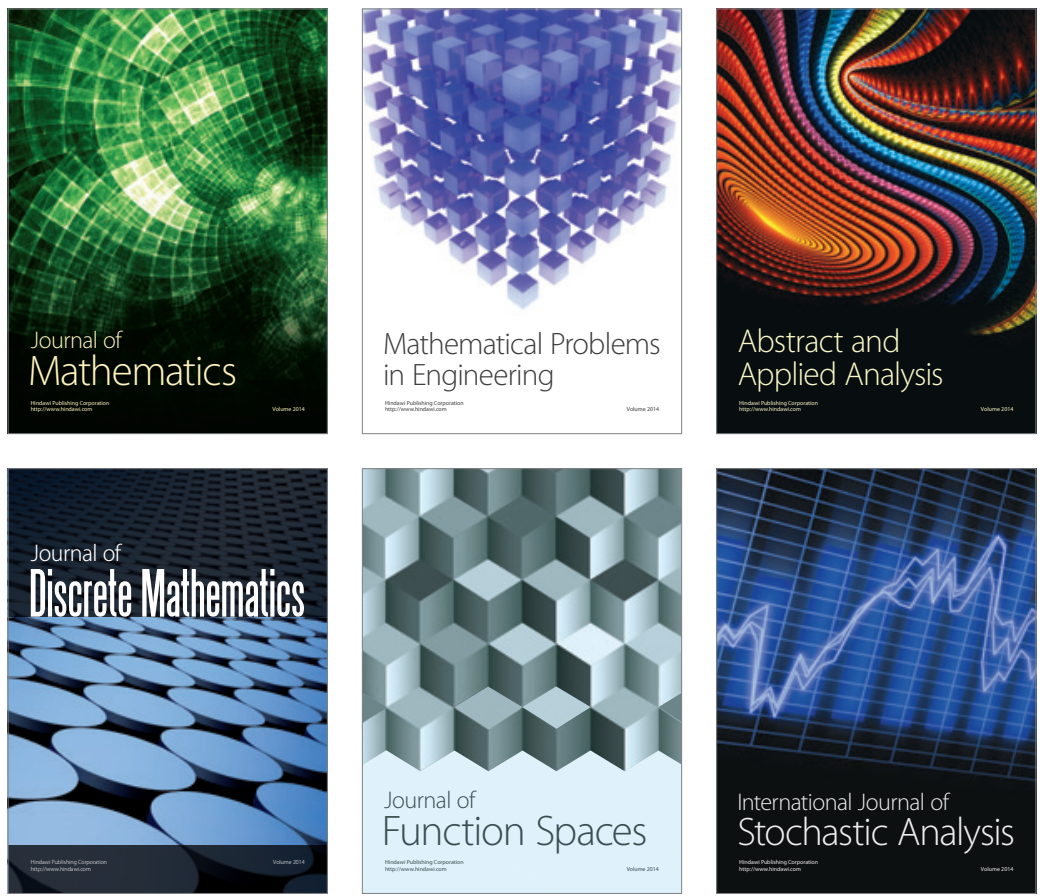

Journal of

Function Spaces

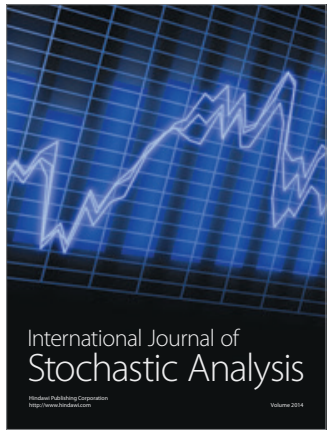

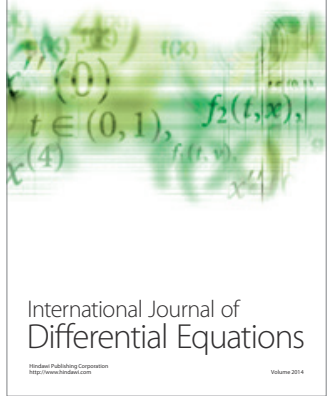
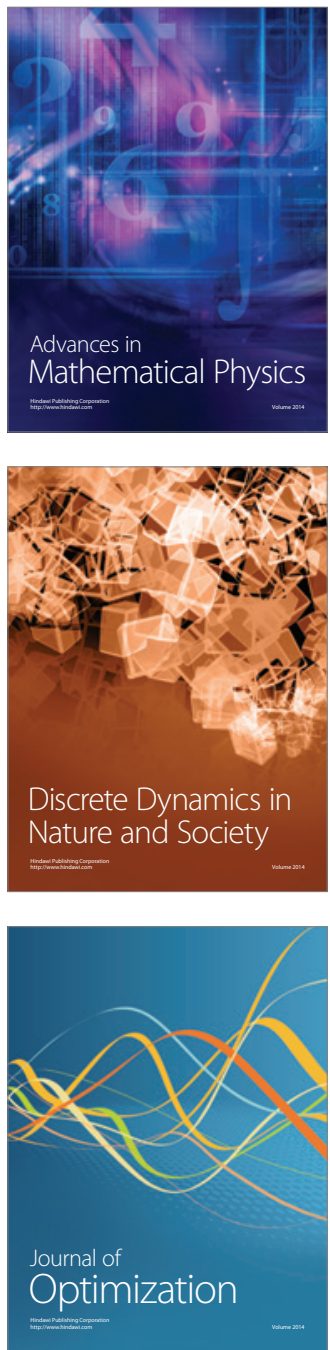